\title{
APOYO EMOCIONAL DE LAS FAMILIAS A LOS PACIENTES EN UNIDADES DE CUIDADOS INTENSIVOS: REVISIÓN BIBLIOGRÁFICA
}

\section{EMOTIONAL SUPPORT OF THE FAMILIES TO THE PATIENTS IN INTENSIVE CARE UNITS: LITERATURE REVIEW}

\author{
Laura Duque Delgado, Encarnación Elena Rincón Elvira, Victoria Eugenia León Gómez
}

\author{
Enfermeras del Servicio Andaluz de Salud
}

DUQUE DELGADO, L., RINCÓN ELVIRA, E., \& LEÓN GÓMEZ, V. (2020). APOYO EMOCIONAL DE LAS FAMILIAS A LOS PACIENTES EN UNIDADES DE CUIDADOS INTENSIVOS: REVISIÓN BIBLIOGRÁFICA.

Revista Ene De Enfermería, 14(3). http://www.ene-enfermeria.org/ojs/index.php/ ENE/article/view/1125 


\section{Resumen}

El aislamiento del paciente crítico con respecto a su familia en la Unidad de Cuidados Intensivos (UCl), tiene repercusiones para ambos, siendo, fundamental el apoyo emocional de los familiares para la recuperación del paciente.

Pudiendo concluir que la $\mathrm{UCl}$ es una de las áreas donde más estrés se genera debido a la cantidad de factores estresantes tanto para los pacientes como para sus familiares. A pesar de esto, las necesidades de la familia se desatienden por parte de los enfermeros/ as, descuidándose a veces el apoyo emocional que estos familiares necesitan cuando su familiar se encuentra ingresado.

Además, la participación de la familia en los cuidados del paciente proporciona apoyo emocional al paciente y disminuye el sufrimiento de ésta, reduciéndose así los síntomas psicológicos que puedan aparecer.

Palabras claves: paciente crítico, enfermera de cuidados críticos, Unidad de Cuidados Intensivos (UCI), familiares del paciente crítico, apoyo emocional.

\section{Abstract}

The isolation of critical patient regarding his family in the Intensive Care Unit (ICU), has implications for both, being fundamental the emotional support of family for patient recovery.

Being able to conclude that the $\mathrm{UCl}$ is one of the areas where more stress is generated due to the amount of stressful factors for the patients and their family. Despite this, the needs of the family are neglected by nurses, neglecting sometimes the emotional support that these relatives need when their relative is admitted.

In addition, the family involvement in patient care provides emotional support to the patient and reduces the suffering of this, thus reducing the psychological symptoms that may appear.

Keywords: critical patient, critical care nurse, Intensive Care Unit (ICU), relatives of critically ill patients, emotional support 


\section{INTRODUCCIÓN}

El propósito de este trabajo se centra en conocer si influye el apoyo emocional de los familiares en los pacientes de Unidades de Cuidados Intensivos (UCI).

En primer lugar y principalmente, debido a la discordancia que existe entre la opinión de distintas enfermeras en la UCI con respecto a la implicación familiar, ya que hay enfermeras que piensan que la familia del paciente crítico puede darle un gran apoyo emocional ocasionando beneficios al paciente, y por lo contrario, también hay enfermeras que piensan que los familiares en esta unidad solo empeoran la situación de su familiar y además, estas enfermeras perciben a la familia como una "carga". Asimismo, al no prestar atención a los familiares, desconocemos cuáles son sus necesidades y esto hace que no podamos implementar un cuidado holístico porque podemos tener desatendidas esas necesidades familiares.

Según describen los textos relacionados con el tema que nos ocupa, el cuidado del paciente crítico es incompleto si no se abordan los problemas de sus familiares (1), por lo que, si las necesidades de los familiares de estos pacientes críticos no son cubiertas, estaremos olvidando el cuidado integral del paciente (2).
El ingreso de una persona en una Unidad de Cuidados Intensivos (UCI), significa una barrera física y psicológica en la vida, tanto para esa persona como para su familia. El aislamiento del enfermo respecto de la familia tiene repercusiones para ambos y de forma indirecta influye también negativamente en el sistema (3). Por lo tanto, la familia es un elemento esencial dentro del proceso de recuperación de todo individuo (4).

En las Unidades de Cuidados Intensivos (UCl), por diferentes motivos se ha mantenido a la familia aislada del paciente. Muchas unidades justifican este hecho basándose en la idea de que los familiares son un riesgo añadido al problema de infección de los pacientes, impiden el descanso e incluso producen alteraciones fisiológicas. Además, algunas enfermeras han argumentado esta restricción, manifestando que la visita interfiere en los cuidados y que la relación continua con la familia produce agotamiento emocional y estrés al paciente (4). Sin embargo, los pacientes expresan que en esta unidad se sienten solos y aislados, y sus familias son un recurso muy importante para aliviar el estrés que les provoca la enfermedad crítica, considerando que es fundamental el apoyo de sus familiares para su recuperación (5).

Pero hoy día, hay enfermeras que no entienden que debemos ofrecer un 
cuidado holístico incluyendo al paciente y a su familia, manteniendo que su trabajo consiste en el cuidado sólo del paciente.

Para poder entender por qué a pesar de las evidencias que contribuyen a que los familiares del paciente crítico requieren una atención a sus necesidades y un horario de visitas flexibles de la $\mathrm{UCl}$, y de los beneficios que existen tanto para los pacientes como para sus familiares, las UCls en España siguen con políticas de visitas restrictivas. Incluso que, durante la visita, la enfermera aproveche para realizar otras tareas, evitándose así, el contacto con las familias (4). Por lo tanto, esta cultura cerrada de las unidades impide que se resuelva la necesidad de proximidad que tienen paciente y familiares, lo que puede crear un empeoramiento en la salud del paciente y su familia. La liberalización de las visitas influye positivamente sobre los pacientes, produciéndose un efecto positivo tanto en su estado emocional como en su recuperación (3).

Los estudios realizados en todo el mundo durante los últimos 30 años indican que las necesidades de los familiares de los pacientes críticos todavía se descuidan, esto requiere un cambio en la práctica clínica (6). Estas necesidades han recibido una atención importante en la investigación dentro del campo de la
Enfermería, han sido bien examinadas mediante el Cuestionario de Necesidades de los Familiares de Pacientes de Cuidados Intensivos (CCFNI), una herramienta de investigación altamente validada que consta de cinco subescalas como son la información, la seguridad, la proximidad, el apoyo y el confort (7).

La enfermera de Cuidados Críticos es el profesional que pasa más horas junto al paciente, y por lo tanto, forma, junto con la familia, un elemento esencial del paciente ingresado en la Unidad de Cuidados Intensivos (8).

Por último, las visitas de los familiares en las Unidades de Cuidados Intensivos son importantes tanto para el paciente como para todos los miembros de la familia (9). A pesar de esto, los familiares y el personal del hospital restringen las visitas de los niños en las Unidades de Cuidados Intensivos (9). Sin embargo, algunos autores encontraron que los guardias de seguridad que permitieron que los niños visitaran a sus familiares, sintieron que la visita era buena para ellos, ya que, a veces denegar a los niños la visita significa dejarlos con dudas y preguntas $(9)$.

Sin embargo, a menudo con el ritmo de trabajo existente en las Unidades de Cuidados Intensivos, la comunicación no es una prioridad. Los familiares esperan que las enfermeras le ofrez- 
can apoyo emocional y que sean empáticas con ellos (10).

Se considera que es esencial el apoyo emocional en las Unidades de Cuidados Intensivos, ya que unas intervenciones adecuadas y efectivas permiten prevenir, eliminar o disminuir todas las alteraciones psicológicas que presentan los pacientes, como pueden ser ansiedad, depresión, alteración de la imagen corporal, temor a la desconocido, dolor, etc y en el caso de los familiares actuar frente la ansiedad, la depresión, los sentimientos de culpa, ayudarles a afrontar una muerte cercana de su familiar, afrontar el duelo, etc (11). De este modo, la intervención psicológica en estas unidades, en ocasiones junto a otros profesionales (como médicos, trabajadores sociales, psicólogos), permite atender al paciente como un ser biopsicosocial (11).

Por lo tanto, el núcleo familiar involucrado requiere tanto de apoyo emocional como de una excelente y clara comunicación sobre el estado de salud del paciente. De allí la importancia del personal de enfermería de brindar un apoyo emocional efectivo que contribuya a mejorar la calidad del cuidado ofertado, unificando criterios al respecto, permitiendo a los profesionales mayor sensibilidad al brindar el cuidado, aspecto que fortalecerá la imagen personal, profesional e institucional (12).

Enlazando con lo anteriormente expuesto, nos planteamos la pregunta de investigación sobre cómo influye el apoyo emocional familiar en los pacientes de Unidades de Cuidados Intensivos.

\section{Metodología}

En primer lugar, se ha realizado una búsqueda bibliográfica del tema de estudio en diferentes bases de datos, las cuales son fuentes de información secundarias. Éstas son nacionales como CUIDEN plus, IME, LILACS, DIALNET plus, PsycINFO e internacionales como PUBMED, CINAHL y SCOPUS.

Hemos empleado estrategias de búsqueda para la misma, mediante los descriptores de Ciencias de la Salud (DeCS) combinados con los distintos operadores booleanos (AND, OR) en las siguientes 8 bases de datos: PUBMED, CINAHL, SCOPUS, CUIDEN plus, LILACS, DIALNET plus, IME y PsycINFO.

Se obtuvieron un total de 59 artículos utilizando como criterios de inclusión los artículos publicados en los últimos 6 años, idioma (inglés y español), texto completo.

Se hizo una primera lectura del título de los artículos. Después, al leer los resúmenes de los 59 artículos, selec- 
cioné 32 artículos y descarté 27 artículos, de los cuales: 2 artículos eran repetidos, 19 artículos diferían del tema a tratar en este trabajo y 6 artículos tenían una muestra insuficiente, menor a 30.

Posteriormente, una vez leídos los 32 artículos completos, he descartado 4 artículos porque la temática no es la que busco según los objetivos plantea- dos y 10 artículos porque han sido utilizados para elaborar la introducción.

Definitivamente, he seleccionado 18 artículos, de los cuales 5 eran estudios cualitativos, 6 eran estudios cuantitativos, 2 eran estudios mixtos y 5 eran revisiones de la literatura.

Las estrategias de búsqueda se describen en la Tabla 1.

Tabla 1 (Estrategias de búsqueda. Elaboración propia)

\begin{tabular}{|c|c|c|c|}
\hline Bases de datos & $\begin{array}{l}\text { Estrategias de búsquedas } \\
\text { (descriptores y booleanos) }\end{array}$ & $\begin{array}{l}\text { Criterios de } \\
\text { inclusión }\end{array}$ & $\begin{array}{l}\text { Artículos } \\
\text { seleccionados }\end{array}$ \\
\hline PUBMED & $\begin{array}{l}\text { · (“ICU patient" OR "critic } \\
\text { patient”) AND family AND } \\
\text { emotions. } \\
\text {. "Intensive Care Unit"” AND } \\
\text { nurs* AND "emotional support" }\end{array}$ & $\begin{array}{l}\text { Full text, English y } \\
\text { Fecha } 2010-2016 \text {. } \\
\text { Full text, English, } \\
\text { Fecha 2010-2016 y } \\
\text { primera lectura del } \\
\text { titulo }\end{array}$ & 10 \\
\hline CINAHL & $\begin{array}{l}\text { "ICU patient"” OR "critical } \\
\text { patient" }{ }^{\star *} \text { AND family AND } \\
\text { emotion* AND nurs* }\end{array}$ & $\begin{array}{l}\text { Full text, English, } \\
\text { all adult, Fecha } \\
2014-2015 \text { y } \\
\text { primera lectura del } \\
\text { titulo. }\end{array}$ & 19 \\
\hline SCOPUS & $\begin{array}{l}\text { (“ICU patient"” OR "critical } \\
\text { patient*") AND family AND } \\
\text { emotion* AND nurs }\end{array}$ & $\begin{array}{l}\text { Date range } \\
2010-2016 \text { y } \\
\text { primera lectura del } \\
\text { título. }\end{array}$ & 5 \\
\hline CUIDEN plus & $\begin{array}{l}\text { (“paciente") AND ((“crit ico") } \\
\text { AND ((“UCl") AND ((“familia") } \\
\text { AND ((“emocional”) AND } \\
\text { (“enfermera"))))) }\end{array}$ & Fecha 2010-2016 & 5 \\
\hline LILACS & $\begin{array}{l}\text { paciente AND UCI AND familia } \\
\text { AND enfermera }\end{array}$ & $\begin{array}{l}\text { Texto completo, } \\
\text { Fecha 2009-2016, } \\
\text { Idioma (English y } \\
\text { español) y artículos } \\
\text { repetidos }\end{array}$ & 3 \\
\hline
\end{tabular}




\begin{tabular}{|c|c|c|c|}
\hline DIALNET plus & $\begin{array}{l}\text { ("ICU patient*" OR "critical } \\
\text { patient"”) AND family AND } \\
\text { emotion* AND nurs* }\end{array}$ & Texto complete & 1 \\
\hline PsycINFO & $\begin{array}{l}\text { ("ICU patient*" OR "critical } \\
\text { patient"”) AND famil" AND } \\
\text { emotion }\end{array}$ & Texto complete & 7 \\
\hline IME & $\begin{array}{l}\text { Paciente critico Y Unidad de } \\
\text { Cuidados Intensivos Y Apoyo } \\
\text { familiar } \\
\text { - Paciente critico Y Unidad de } \\
\text { Cuidados Intensivos }\end{array}$ & $\begin{array}{l}\text { Texto completo } \\
\text { Texto completo, } \\
\text { año publicación } \\
\text { después de } 2009 \text { y } \\
\text { lectura del título }\end{array}$ & 1 \\
\hline
\end{tabular}

\section{REsultados}

En el Diagrama de Flujo (Figura 2) se especifica la descripción del proce- so de selección de los artículos revisados con la aplicación de los criterios de selección y exclusión.

Figura 2. Flujograma.

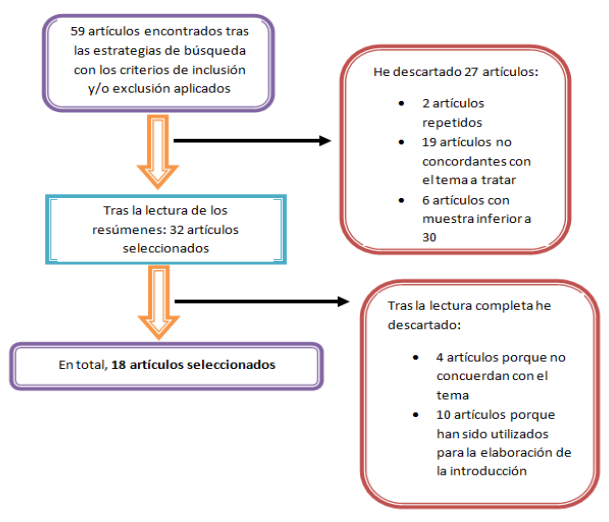

En la tabla 3 se realiza una síntesis de los artículos utilizados para dar respuesta a los objetivos planteados en nuestro estudio, en la cual se clasifican mostrando título, autor/es, año de publicación y revista, tipo de estudio y resultados principales. 


\section{DISCUSIÓN}

Se han analizado los artículos y se ha desarrollado la discusión, respondiendo a los distintos objetivos propuestos.

Factores estresantes en la relación familia-paciente en las Unidades de Cuidados Críticos:

A. García Mozo et al en el año 2010 llegaron a un amplio consenso acerca de cuáles son los factores que generan estrés en los familiares de los pacientes críticos ingresados en una Unidad de Cuidados Críticos, éstos se dividen en los referidos a las características de la $\mathrm{UCl}$ y los referidos a los familiares. Con respecto a los referidos a las características de la UCI, están (13):

- Tecnificación del entorno

- Falta de intimidad y de espacios adecuados

- Alto número de profesionales diferentes que atienden al paciente

- Falta de confianza en los servicios de salud

- Lenguaje tecnificado e información escasa y sometida a las normas restrictivas de la unidad

- Síndrome de estrés del traslado: traslado de la $\mathrm{UCl}$ a una unidad de hospitalización.

Con respecto a los referidos a los familiares, están (13):

- Situación de crisis vital y de muerte inminente

- Falta de control sobre la situación

- Incertidumbre en los momentos de espera y sobre el futuro del paciente

- Cambio de roles en el seno de la familia: el ingreso en una unidad de críticos provoca un desajuste en su funcionamiento

- No sentirse preparados para tomar decisiones sobre el paciente

- Percepción de falta de libertad para verbalizar dudas y temores (sobrecarga de trabajo de las enfermeras)

Sin embargo, Van Mol et al en 2014 , en su estudio sobre las perspectivas de los familiares en la calidad de los cuidados en una Unidad de Cuidados Intensivos, coincide con A. García Mozo et al, señalando que el ambiente de esta 
unidad es estresante debido a la multitud de aparatos desconocidos, al personal y a otros pacientes que pueden influir en el nivel de estrés (13,14) pero, además, Van Mol et al indican que los sonidos y los olores también son factores estresantes (14).

Por otro lado, Alham Abuatiq en el año 2015 en su estudio sobre la percepción de estrés de los pacientes y los proveedores de salud señala que la presencia de la familia con los pacientes críticos durante su estancia en la Unidad de Cuidados Intensivos tiene un impacto mayor en su puntuación sobre el estrés, los pacientes que tienen un miembro de la familia presente con ellos durante su enfermedad tienen puntuaciones de estrés significativamente menores que aquellos que no tenían a los familiares (15). Además, este autor indica que el factor estresante "tener dolor" se clasifica como el más estresante para los pacientes, seguido por "no ser capaz de comunicarse" "no tener el control de sí mismo" y, por último, "preocupaciones financieras" (15).

Por el contrario, Verceles et al en 2014 afirman en su estudio que la dificultad para dormir ha sido puntuada como uno de los principales factores de estrés de los pacientes críticos y sus familias, ya que los trastornos del sueño y el cansancio han sido identificados como las respuestas fisiológicas y de comporta- miento al estrés que ocasiona tener un miembro de la familia en la Unidad de Cuidados Intensivos (16).

También refieren estos autores que las directrices para el apoyo de los familiares de los pacientes críticos se han centrado en la mejora de la comunicación y la satisfacción de las necesidades espirituales y emocionales de los miembros de la familia, como estrategias para disminuir el estrés que provoca el ingreso de un familiar en la $\mathrm{UCl}$, pero el apoyo a los miembros de la familia también tiene que incluir intervenciones para aliviar la privación del sueño, como facilitar zonas adecuadas a la familia que puedan aliviar su estrés, zonas tranquilas para poder descansar y dormir, 0 educar a los familiares sobre la importancia de tener un sueño adecuado y fijo mediante la asignación de un horario dedicado a dormir (16).

Necesidades que pueden encontrarse en los familiares de los pacientes en las Unidades de Cuidados Críticos:

Según Pardavila y Vivar en su revisión bibliográfica, en el año 2012, sobre las necesidades de la familia en las Unidades de Cuidados Intensivos, señalan que diversos estudios han indicado que la necesidad de información es 
la de mayor importancia para las familias. También advierte que es importante una adecuada coordinación entre la información médica y enfermera para que los familiares reciban la mejor información posible (17). Asimismo, Lee and Lau en 2003, Chiu et al en 2004, Al-Mutair et al en 2013, Khalaila en 2013, identificaron prioritarias además de la necesidad de información, la necesidad de proximidad y de confianza. Por el contrario, Bijttebier et al 2001, Holden et al 2002, Verhaeghe et al 2005 identifican las necesidades de comodidad y de apoyo como menos importantes (18).

Plakas et al, en el año 2009, en su estudio sobre las necesidades de la familia indican que los familiares de los pacientes críticos padecen emociones positivas, relacionadas con la esperanza de mejoría de su familiar, y emociones negativas producidas por el miedo a perder a su ser querido, éstas pueden provocar una fuerte sensación de impotencia y de incapacidad para ayudar a su ser querido. Debido a esto, el apoyo emocional a las familias por parte de los/ as enfermeros/as es un aspecto importante que influye en su bienestar. Además, estos autores confirman con la evidencia que con frecuencia las enfermeras descuidan las necesidades emocionales de los familiares debido a que no realizan los cuidados necesarios para cubrirlas. Al mismo tiempo, Henneman y Cardin, en 2002, demandan que los familiares necesitan un mayor apoyo emocional cuando su familiar está en la UCl, al encontrarse en un estado de vulnerabilidad y de inquietud (17).

Los autores A. García Mozo et al en el año 2010 en su estudio sobre el desarrollo de una guía de atención a los familiares del paciente crítico indican que existe un desajuste entre las necesidades de los familiares de la UCI y los cuidados que reciben por parte de las enfermeras (13), esto también está apoyado por los autores C. Zaforteza Lallemand et al en su estudio sobre la mejora del cuidado a los familiares del paciente crítico en el año 2010 (19).

Debido a este desajuste, y para aumentar el conocimiento de los profesionales de la $\mathrm{UCl}$ acerca de la experiencia que atraviesan los familiares en esta unidad y así poder establecer unos planes de cuidados para trabajar con ellos, A. García Mozo et al, diseñan una guía de atención a las necesidades de los pacientes y de los familiares de éstos para que exista un acuerdo sobre cómo atender a los familiares de la UCI. La creación de esta guía surge durante los años 2007-2009 mediante el proyecto llamado Promoción de los cuidados dirigidos a los familiares del paciente crítico. A lo largo de este proceso se priorizaron 
cuatro propuestas para mejorar los cuidados a los familiares: elaboración de un póster informativo para familias (para mejorar las condiciones de espera al ingreso), desarrollo de un plan específico de formación continuada sobre las habilidades sociales, estrategias de comunicación y manejo de situaciones de contenido emocional (para dotar a los profesionales de herramientas para manejar el estrés y la comunicación), ampliación de los horarios de visita (con el fin de facilitar la cercanía familia-paciente) y elaboración de una guía de atención a las familias (con el objeto de establecer un "acuerdo de mínimos" de atención a los familiares) $(13,20)$.

Como bien apuntan Pardavila y Vivar, en el año 2012, en su revisión bibliográfica sobre las necesidades de los familiares de la $\mathrm{UCl}$, el tener a un ser querido ingresado en la $\mathrm{UCI}$ afecta a los roles familiares, a las relaciones y a las responsabilidades del resto de los miembros de la familia, y esto desencadena, la interrupción de los procesos familiares. La proximidad, es decir, estar cerca del ser querido y verlo con regularidad, o incluso estar continuamente a su lado, es importante para el entorno social, esto lleva a los familiares a manifestar su disconformidad con la duración y el horario de visitas de la unidad y a expresar su deseo de unos horarios más flexibles, la posibilidad éste ayudaría a los familiares a sentirse partícipes en el cuidado de su familiar. Por lo que, una de las primordiales necesidades emocionales manifestadas por los familiares compete a la necesidad de proximidad con su ser querido. Las familias que se encuentran alejadas de su familiar presentan mayor ansiedad a diferencia de aquellas que pueden estar cerca del paciente (17). Igualmente, Rodríguez et al, en el año 2003, en su estudio sobre la implicación familiar en los cuidados del paciente crítico, indican que las visitas abiertas ayudan a disminuir los niveles de ansiedad y aumentan el nivel de satisfacción de los familiares, por el contrario, Hardicre, en el año 2003, en su estudio sobre la satisfacción de las necesidades de las familias de los pacientes en Unidades de Cuidados Intensivos destaca que la visita prolongada puede conllevar la pérdida de identidad del familiar y un abandono de sus propias necesidades (17).

Por otro lado, Karlsson et al, en el año 2011, en su estudio sobre la satisfacción de los familiares con el cuidado recibido en Cuidados Intensivos, determina que el punto con el que las familias están más insatisfechas es con las salas de espera, este resultado revela lo importante que es la confortabilidad y la cercanía a la UCI de las salas de espera. 
Los autores Pardavila y Vivar, en su estudio comentado anteriormente, también revelan que los familiares valoran que las salas de espera son un lugar pequeño, frío, confuso, incómodo, con poca intimidad, aterrador y donde se debe estar alerta. De vez en cuando, las salas de esperas favorecen el soporte emocional entre familias que están viviendo situaciones parecidas. Todo esto, ha encaminado a Álvarez y Kirby en 2006, en su estudio sobre la perspectiva de las necesidades de las familias del paciente crítico a señalar la importancia de prestar atención a los pequeños detalles de las salas de espera, por ejemplo, la disponibilidad de un teléfono a disposición de los familiares, ya que éstas pueden fomentar la comodidad de las familias en situaciones de gran vulnerabilidad y así aliviar su angustia (18).

Por el contrario, los autores C. Zaforteza Lallemand et al en 2010 en su estudio sobre la mejora del cuidado a los familiares del paciente crítico, manifiestan que el cuidado técnico del paciente en la $\mathrm{UCl}$ ocasiona que otros aspectos del proceso, como puede ser la atención a los familiares, no se tengan en cuenta o no sean percibidos como una prioridad, de manera que los profesionales de cuidados intensivos asumen que el valor de su trabajo radica en la "efectividad tecnológica", causando que los/as enfermeros/ as subestimen la vivencia de los familiares. Para promover un cambio para mejorar los cuidados de los familiares se estableció un proceso y se determinaron áreas susceptibles de mejora como son: el proceso de información a las familias, el abordaje de los procesos de duelo de los familiares, la limitación del esfuerzo terapéutico, la protección de la intimidad y la política de visitas, con respecto a ésta, existe diversidad entre los profesionales, ya que un grupo no considera a los familiares como un objetivo de atención, y el otro grupo sí (19).

Concuerdan Holden et al 2002, Verhaeghe et al 2005, Maxwell et al 2007 en sus estudios, que el personal de la unidad casi siempre no cumple con las necesidades de estos familiares (18) y Bijttebier et al 2001, Omari de 2009, AlMutair et al 2013 en sus estudios exponen que algunos investigadores han encontrado que las necesidades de cercanía, de información y de confianza eran frecuentemente las más insatisfechas, mientras que Lee y Lau en 2003, Browning y Warren en 2006 han encontrado que esas necesidades de cercanía, de información y de confianza eran las más satisfechas al contrario de las necesidades de apoyo y comodidad que eran las necesidades más satisfechas con menos frecuencia (18). 
Y en esta misma dirección, los autores E.B. Carlson et al en 2015 en su estudio sobre el cuidado en la Unidad de Cuidados Intensivos manifiestan que los miembros de la familia en general expresan satisfacción con la atención recibida en esa unidad, pero las puntuaciones relativamente más bajas se dieron para la necesidad de comunicación, de información y de apoyo emocional, entre un $17 \%$ y un $20 \%$ de sus participantes (21).

\section{Problemas de salud que pue-} den aparecer en los familiares de los pacientes en las Unidades de Cuidados Críticos:

Los autores Pardavila y Vivar en 2012 en su estudio sobre las necesidades de la familia en las Unidades de Cuidados Intensivos (UCI), indican que la falta de una comunicación efectiva con las familias del paciente crítico provoca en los familiares sentimientos de preocupación, ansiedad, frustración, soledad, desánimo, enfado, tristeza y miedo, por eso, para aliviar este sufrimiento de las familias, los autores Álvarez y Kirby en el año 2006 reivindicaron la importancia de proporcionar una información clara, oportuna y coherente que les ofrezca una orientación para mantener la confianza en la recuperación de su familiar (17).
En la misma línea que los autores Pardavila y Vivar en 2012, Lee y Lau en 2003, Auerbach et al en 2005 y Davidson en 2009, en sus estudios, confirman que el ingreso de un familiar en una Unidad de Cuidados Intensivos es frecuentemente inesperado, provocando síntomas psicológicos en los miembros de la familia como shock, culpa, confusión, estrés, depresión, cansancio, miedo, preocupación y ansiedad (18). Igualmente, Carlson et al en 2015 en su estudio sobre el cuidado en la $\mathrm{UCl}$, así como los síntomas anteriores, añade el trastorno de estrés postraumático (20).

Además, Van Mol et al en 2014 en su estudio sobre las perspectivas de los familiares en la calidad de los cuidados en una $\mathrm{UCl}$ corrobora que el ingreso de un familiar puede hacer que en la familia aparezca los síntomas anteriores, incluso incorpora que se puede llegar a desarrollar lo que se conoce como "Síndrome post-UCI de la familia" (15).

Verceles et al en 2014 certifican en su estudio que existe un exceso de somnolencia diurna entre los miembros de la familia de los enfermos críticos, ya que más de la mitad de los familiares $(50,2 \%)$ presentan somnolencia diurna, incluso más que la población general (10-20\%). En este estudio la somnolencia diurna excesiva y la funcionalidad disminuida se localizaron en toda la po- 

APOYO EMOCIONAL DE LAS FAMILIAS A LOS PACIENTES EN UNIDADES DE CUIDADOS INTENSIVOS

blación de estudio (225 miembros de familia). Estos familiares también sufrieron una disminución significativa en la calidad de vida debido a la falta de sueño, viéndose afectada la productividad general, que incluye la concentración, la realización de tareas y quehaceres diarios, los recordatorios, el trabajo de las aficiones, los asuntos financieros, las tareas de empleo y el mantenimiento de conversaciones telefónica. En este estudio también se demuestra que hasta un $80 \%$ de los miembros de la familia tienen menos sueño, el $70 \%$ sufren una peor calidad del sueño, y el $20 \%$ necesitan medicamentos hipnóticos después de que su miembro de la familia ingresara en la $\mathrm{UCl}(16)$.

\section{Beneficios de la comunicación} paciente-familia, participación de la familia en los cuidados y el papel de la enfermera en esa integración:

La comunicación en la Unidad de Cuidados Intensivos es un reto debido a la complejidad, a la gravedad del paciente, a la incertidumbre y a las cuestiones éticas, además, como bien dice la autora Grant en su estudio en 2015 sobre la resolución de los problemas de comunicación en la $\mathrm{UCl}$, las familias están más insatisfechas con la comunicación en esta unidad que con cualquier otro tema de atención (22). Esto también está apoyado por Carlson et al en su estudio del 2015 que además de la insatisfacción con la comunicación, también añaden la insatisfacción con el apoyo emocional. Por lo que, estos últimos autores declaran que la mejora de la comprensión y la comunicación entre los miembros de la familia y los pacientes puede ayudar a reducir los sentimientos de angustia, la incertidumbre sobre el estado del paciente y la necesidad de apoyo emocional. Asimismo, estos manifiestan que el apoyo emocional para los familiares también se puede aumentar mediante el fomento del apoyo mutuo entre las familias de los pacientes de la UCI (21).

Los autores Achury Saldaña et al en 2014 en su estudio sobre el proceso de comunicación entre los profesionales de enfermería y los pacientes en estado crítico, han determinado que un $91,6 \%$ de los/as enfermeros/as consideran importante la comunicación con los pacientes y sus familias, debido a esto un $75,8 \%$ tienen como objetivo llevar a cabo actividades para fomentar la comunicación con paciente-familia, a su vez, el $86,6 \%$ confirma que habla con los pacientes entre 2 y 4 veces por turno, mientras que un $70,5 \%$ solo se comunica con las familias 1 ó 2 veces por turno. Además, sólo el $53,7 \%$ de los/as enfermeros/as integran sus emociones en la 

APOYO EMOCIONAL DE LAS FAMILIAS A LOS PACIENTES EN UNIDADES DE CUIDADOS INTENSIVOS

atención al paciente. Este autor revela que el proceso de comunicación entre las enfermeras y los pacientes de la $\mathrm{UCl}$ está limitado por las políticas de visitas restrictivas y por la escasa formación académica de los/as enfermeros/as para apoyarlos emocionalmente (23). Sin embargo, Shaw et al en 2013 en su estudio sobre la formación del equipo multidisciplinario para mejorar la comunicación familiar en la $\mathrm{UCI}$, han tratado de capacitar al equipo que allí trabaja sobre la comunicación con las familias de los pacientes críticos. Para ello han llevado a cabo una sesión de entrenamiento interactivo de 90 minutos con una simulación de un caso y una sesión informativa, involucrando a todos los miembros de la familia. Esto, mejoró la satisfacción familiar de la $\mathrm{UCl}$, mejorando así la confianza de la comunicación con los profesionales (24). Asimismo, Brysiewicz et al en 2010 en su estudio sobre las experiencias de las enfermeras en la prestación de apoyo psicosocial a los familiares de los pacientes críticos en $\mathrm{UCl}$, manifiestan que la mayoría de los/as enfermeros/as sienten que cuatro factores principales afectan en el apoyo psicosocial que pueden ofrecer, éstos son la diversidad cultural, las barreras en la comunicación, los recursos y la formación insuficientes (25).

Por otro lado, Carlson et al en 2015 en su estudio sobre el cuidado en la $\mathrm{UCl}$, comentan que un método adecuado para mejorar la atención y la satisfacción general con la atención en la $\mathrm{UCl}$, es aumentar la participación de los miembros de la familia en el cuidado de su familiar, ya que cuando las familias ayudan con el cuidado, los pacientes se benefician a través de una mayor orientación, humanización, apoyo emocional; y los familiares se benefician en la reducción de la incertidumbre y el temor por el estado del paciente (21).

En esta misma tendencia, Marco Landa et al en su estudio sobre las creencias y actitudes de las enfermeras de Cuidados Intensivos sobre el efecto que la visita abierta produce en el paciente, familia y enfermeras, ya revelaban por el año 2000 que tras ampliar las visitas de las $\mathrm{UCl}$, que de ser restrictivas, habían pasado a ser "abiertas", y permanecer los familiares periodos de tiempo con su familiar, flexibilizando el horario según las necesidades del paciente y su familia; el $93 \%$ de los/as enfermeros/as se manifestaban satisfechos con las nuevas normas y todos opinaron que la familia proporciona apoyo emocional al paciente, minimizando su aburrimiento $y$, el $91 \%$ opinaron que incrementaban su deseo de vivir. Igualmente, el $100 \%$ creía que aumentaba la satisfacción de la familia, el $93 \%$ que disminuía la ansiedad de ésta, proporcionando así un mayor 
apoyo al equipo de la UCl y mayor seguridad de que el paciente estaba recibiendo el mejor cuidado posible (26).

En la misma línea que el anterior estudio, Rodríguez et al en 2003 en su estudio sobre la implicación familiar en los cuidados del paciente crítico, publicó los resultados obtenidos en la $\mathrm{UCl}$ del Hospital de Valme, concluyendo resultados prácticamente idénticos a los reflejados en el estudio de Marco Landa et al. Por el contrario, el $85 \%$ de los/as enfermeros/s en el estudio de Marco Landa et al, y el $61 \%$ en el de Rodríguez et al opinaban que la presencia de los familiares interrumpía los cuidados de enfermería (26).

Asimismo, Kean y Mitchell en 2013 en su estudio sobre la percepción de las enfermeras de Cuidados Intensivos con respecto a las familias, afirman que las enfermeras opinan que invitar a los familiares a formar parte de la atención del paciente, debe ser una práctica habitual en la $\mathrm{UCl}$, ya que los miembros de la familia son el recurso para el cuidado de estos pacientes durante una trayectoria de recuperación a menudo prolongada, incluso manifiestan que la participación de las familias en los cuidados del paciente de la $\mathrm{UCl}$, hace que las enfermeras tengan la oportunidad de proporcionar el apoyo y la continuidad que tanto los familiares como el paciente necesitan durante el periodo de la enfermedad crítica, aparte de esto, esta implicación satisface mejor las necesidades de las familias y favorece la comunicación (27). Por el contrario, McConnell y Moroney en 2015 en su estudio sobre los desafíos de la enfermería en Cuidados Críticos, con respecto a la involucración de los familiares en el cuidado del paciente, refieren que las enfermeras de Cuidados Intensivos podrían involucrar a los familiares en las actividades de cuidado que no impacten en la privacidad del paciente pero que existen barreras como el papel paternalista de las enfermeras, las políticas de visita de la UCl y/ o las diferentes opiniones de las enfermeras de $\mathrm{UCl}$ con respecto a la participación de la familia (28). En el mismo eje que estos anteriores estudios, Zaforteza Lallemand et al en 2009, exponen que de 93 enfermeros que participaron en el estudio, 51 no estaban de acuerdo con la apertura de la $\mathrm{UCl}$ frente a 42 que si estaban de acuerdo (20).

Por último, Garrouste-Orgeas et al en 2014 y Ullman et al en 2014 en sus estudios ponen de manifiesto los beneficios de los diarios de la UCI $(29,30)$. Garrouste-Orgeas et al exponen que los diarios tienen efectos positivos sobre los miembros de la família, ya que éstos los utilizan para acceder, entender y asimilar la información sobre el estado de salud 
de su familiar, para compartir esta información con los otros miembros de la familia, además, permiten una conexión con el paciente y los enfermeros/as, humanizando el ambiente de la UCI (29). Sin embargo, Ullman et al en su estudio declaran que no hay evidencia mínima de los beneficios que estos diarios para los pacientes y sus familiares, pero si se puede afirmar que éstos reducen la sintomatología de estrés postraumático en los familiares (30).

\section{LIMITACIONES DEL PROCESO DE REVISIÓN}

Con la realización de esta revisión bibliográfica, la principal limitación que nos hemos encontrado es la escasa literatura científica que existe en las bases de datos sobre el apoyo emocional que los familiares les pueden dar a los pacientes en las Unidades de Cuidados Intensivos en España, ya que aquí la mayoría de las UCls siguen con una política de visitas restrictivas y como consecuencia de esto, no se puede trabajar con la familia, lo que hace que no se lleven a cabo estudios con los familiares de los pacientes críticos en España.

\section{CONCLUSIONES}

1. La Unidad de Cuidados Intensivos (UCl) es una de las áreas del Hospital donde más estrés se genera, tanto en los pacientes críticos ingresados en ella como en los miembros de su familia. Los pacientes que tienen el apoyo de su familia durante la estancia en esta unidad tienen menor puntuación en el nivel de estrés que aquellos pacientes que no tienen a sus familiares con ellos.

2. Las necesidades de los familiares de los pacientes ingresados en las Unidades de Cuidados Intensivos más importantes son las necesidades emocionales, la necesidad de ser informados, la necesidad de proximidad y la de seguridad. Estas necesidades, son descuidadas a menudo por parte de los/as enfermeros/as, encontrándose un desajuste entre las necesidades de los familiares y los cuidados que reciben en esta unidad. El apoyo emocional por parte de los/as enfermeros/as a la familia, es un aspecto fundamental que contribuye al bienestar de ésta, en situación de vulnerabilidad, cuando su familiar se encuentra ingresado en la UCI.

3. A pesar de que la necesidad de proximidad se considera primordial, la 
mayoría de las UCls en España siguen con una política de visitas restrictivas. Existen diferencias entre los distintos enfermeros/as al considerar a los familiares o no, como objeto de atención.

4. En los familiares de los pacientes críticos hospitalizados en las Unidades de Cuidados Intensivos existe un sufrimiento permanente que desencadena diversos síntomas psicológicos, destacando trastorno de estrés postraumático, somnolencia diurna, disminución de la calidad de vida y el síndrome post-UCI de la familia. Éstos se deben a la falta de comunicación efectiva, por lo que, mejorando la comunicación entre paciente-familia se podrían reducir estos síntomas psicológicos de los familiares.

5. La mayoría de los/as enfermeros/as consideran que la comunicación en la UCl está limitada por la diversidad cultural, las barreras en la comunicación (política de visitas restrictiva), los recursos insuficientes y la escasa formación académica.

6. Una gran parte de los/as enfermeros/as piensan que debe fomentarse la participación de los familiares en los cuidados del paciente ya que esto reduce los síntomas psicológicos de los familiares y supone un recurso importante para la recuperación del paciente; frente a una minoría de enfermeros/as que piensan que la participación de la familia interrumpe los cuidados del paciente.

7. Por último, los diarios utilizados en la $\mathrm{UCI}$ reducen la sintomatología psicológica de las familias y mejora la conexión con el paciente-familia y los/ as enfermeros/as. 


\section{BIBLIOGRAFÍA}

1. Campo-Martínez MC, Cotrina-Gamboa MJ. Relación de ayuda al familiar del paciente en situación crítica. Enfermería Glob. 2011;10(24):103-9.

2 Llamas-Sánchez F, Quesada-Pérez MT, Guzmán-Valencia T. Protocolo de información a familiares de pacientes ingresados en Unidad de Cuidados Intensivos. Hospital Universitario Virgen Macarena. Servicio Andaluz de Salud. 2012.

3 Martínez M., Rodríguez F, Rodríguez A, Roncero M., Morgado J, Theodor L., et al. Implicación familiar en los cuidados del paciente crítico. Enfermería Intensiva [Internet]. 2014;14(3):96-108. Available from: http://www.elsevierinstituciones.com/ficheros/ p d f l 142/142v14n03a13051385pdf001.pdf\#page =1\&zo om=auto,-107,799

4 Vázquez-Calatayud M, Eseverri-Azcoiti MC. El cuidado de las familias en las unidades de cuidados intensivos desde la perspectiva de Jean Watson. Enferm Intensiva. 2010. p. 161-4.

5 Gooding T, Pierce B, Flaherty K. Partnering with family members to improve the intensive care unit experience. Crit Care Nurs Q [Internet]. 2012;35(3):216-22. Available from: http://www.ncbi.nlm.nih.gov/pubmed/22668993

6 Khalaila R. Patients' family satisfaction with needs met at the medical intensive care unit. Journal Of Advanced Nursing [serial on the Internet]. (2013, May), [cited March 23, 2016]; 69(5): 1172-1182 11p. Available from: CINAHL Complete.

7 Al-Mutair AS, Plummer V, Clerehan R, O'Brien AT. Families' needs of critical care Muslim patients in Saudi Arabia: A quantitative study. Nurs Crit Care. 2014;19(4):185-95.

8 Gálvez-González M. Cuando la familia lo vive, las palabras sobran. El modelo de cuidados compartidos en la fase terminal del paciente crítico : When family experience it, words are not necessary. The model of shared cares in the terminal phase of the critic patient. Archivos De La Memoria [serial on the Internet]. (2011), [cited March 23, 2016]; (. 8): 3. Available from: Dialnet Plus.

9 Knutsson S, Bergbom I. Children's thoughts and feelings related to visiting critically ill relatives in an adult ICU: A qualitative study. Intensive Crit Care Nurs [Internet]. 2016;32:33-41. Available from: http://dx.doi.org/10.1016/j.iccn. 2015.07.007

10 Siddiqui S, Sheikh F, Kamal R. "What families want - an assessment of family expectations in the ICU". Int Arch Med. 2011;4:21.

11 Álvarez J, Artigas A, Belda F.J., Bonet B, Caparrós T, Espino M, Gil J, Murillo F, Sanz M.A. Trata- do de Cuidados Críticos y Emergencias. $2^{\mathrm{a}}$ ed. Madrid: Arán; 2002.

12 Bautista Rodríguez LM, Arias Velandia MF, Carreño Leiva ZO. Percepción de los familiares de pacientes críticos hospitalizados respecto a la comunicación y apoyo emocional. Revista CUIDARTE. 2016;7(2):1297.

13 García-Mozo A, Sánchez-Roldán F, AmorósCerdá SM, Balaguer-Albarracín F, Díez-Alcalde M, Durán-Torres MT, et al. Desarrollo de una guía de atención a los familiares del paciente crítico. Enferm Intensiva. 2010;21(1):20-7.

14 Van Mol MMC, Bakker EC, Nijkamp MD, Kompanje EJO, Bakker J, Verharen L. Relatives' perspectives on the quality of care in an Intensive Care Unit: The theoretical concept of a new tool. Patient Educ Couns. 2014;95(3):406-13.

15 Abuatiq A. Patients' and Health Care Providers' Perception of Stressors in the Intensive Care Units. Dimens Crit Care Nurs [Internet]. 2015;34(4):205-14 10p. Available from: http:// search.ebscohost.com/login.aspx? direct $=$ true $\& d b=j l h \& A N=109798488 \&$ site $=$ ehost live

16 Verceles AC, Corwin DS, Afshar M, Friedman EB, McCurdy MT, Shanholtz C, et al. Half of the family members of critically ill patients experience excessive daytime sleepiness. Intensive Care Med. 2014;40(8):1124-31.

17 Pardavila-Belio MI, Vivar CG. Necesidades de la familia en las unidades de cuidados intensivos.Revisión de la literatura. Enferm Intensiva [Internet]. 2012;23(2):51-67. Available from: www.elsevier.es/ei

18 Khalaila R. Meeting the needs of patients' families in intensive care units. Nurs Stand. 2014;28(43):37-44.

19 Zaforteza-Lallemand C, Prieto-González S, Canet-Ferrer TP, Díaz-López Y, Molina-Santiago $\mathrm{M}$, Moreno-Mulet $\mathrm{C}$, et al. Mejorando el cuidado a los familiares del paciente crítico: estrategias consensuadas. Enferm Intensiva. 2010;21(1):119.

20 Zaforteza-Lallemand C, García-Mozo A, Quintana-Carbonero R, Sánchez-Calvín C, AbadíaOrtiz E, Albert-Miró-Gayà J. Abrir la unidad de cuidados intensivos a los familiares: ¿Qué opinan los profesionales? Enferm Intensiva. 2010;21(2):527

21 Carlson EB, Spain DA, Muhtadie L, McDadeMontez L, Macia KS. Care and caring in the intensive care unit: Family members' distress and perceptions about staff skills, communication, and emotional support. J Crit Care. 2015;30(3):55761.

22 Grant M. Resolving communication challenges in the intensive care unit. AACN Adv Crit Care. 26(2):123-30. 
23 Achury-Saldaña DM, Pinilla-Alarcón M, Alvarado-Romero $\mathrm{H}$. Aspects that facilitate or interfere in the communication process between nursing professionals and patients in critical state. Investig Educ en Enferm [Internet]. 2015;33(1):102-11 10p. Available from: http:// search.ebscohost.com/login.aspx? direct $=$ true $\& d b=c c m \& A N=109819073 \&$ site $=$ ehost live\&scope $=$ site
$24 \quad$ S h a$$
J,
$$
D a vidson

E, Smilde I, Sondoozi T, Agan D. Multidisciplinary team training to enhance family communication in the ICU. Crit Care Med [Internet]. 2014;42(2):265. Available from: www.ccmjournal.org

25 Brysiewicz $P$, Bhengu $B$. The experiences of nurses in providing psychosocial support to families of critically ill trauma patients in intensive care units A study in the Durban metropolitan area [Internet]. Southern African Journal of Critical Care. 2010. p. 42-51. Available from: http://www.ajol.info/ index.php/sajcc/article/view/70113

26 Arnuedo JMN. Revisión de los estudios sobre profesionales de enfermería de las Unidades de Cuidados Intensivos de España. Enfermería Glob [Internet]. 2012;26(1695-6141):267-89. Available from: http://scielo.isciii.es/pdf/eg/v11n26/ revision1.pdf

27 Kean S, Mitchell M. How do intensive care nurses perceive families in intensive care? Insights from the United Kingdom and Australia. J Clin Nurs. 2014;23(5-6):663-72.

28 Mcconnell B, Moroney T. Involving relatives in ICU patient care: Critical care nursing challenges. $\mathrm{J}$ Clin Nurs. 2015;24(7-8):991-8.

29

Garrouste Orgeas M, Périer A, Mouricou P, Grégoire C, Bruel $C$, Brochon $S$, et al. Writing in and reading ICU diaries: Qualitative study of families' experience in the ICU. PLoS One. 2014;9(10).

30 Ullman AJ, Aitken LM, Rattray J, Kenardy J, Le Brocque R, MacGillivray $S$, et al. Diaries for recovery from critical illness. The Cochrane database of systematic reviews. 2014. 
ANEXOS

Tabla 3. Síntesis de resultados

\begin{tabular}{|c|c|c|c|c|c|}
\hline $\begin{array}{l}\text { TITULO } \\
\text { DEL } \\
\text { ARTICULO }\end{array}$ & AUTORES & AÑO & REVISTA & DISEÑO & $\begin{array}{l}\text { PRINCIPALES RESULTADOS/ } \\
\text { CONCLUSIONES }\end{array}$ \\
\hline $\begin{array}{l}\text { Necesidade } \\
\text { s de la } \\
\text { familia en } \\
\text { las } \\
\text { unidades de } \\
\text { cuidados } \\
\text { intensivos. } \\
\text { Revisión de } \\
\text { la literatura }\end{array}$ & $\begin{array}{l}\text { M.I. Pardavila } \\
\text { Belio y } \\
\text { C.G. Vivar }\end{array}$ & 2012 & $\begin{array}{l}\text { Enfermer } \\
\text { ía } \\
\text { intensiva }\end{array}$ & $\begin{array}{l}\text { Revisión } \\
\text { de la } \\
\text { literatura }\end{array}$ & $\begin{array}{l}\text { Se han identificado cuatro grupos de } \\
\text { necesidades en las familias durante el } \\
\text { ingreso del paciente en UCI: cognitiva, } \\
\text { emocional, social y práctica. Las } \\
\text { enfermeras de Cuidados Intensivos } \\
\text { deben identificarlas para poder así } \\
\text { prestar una atención de ayuda, apoyo } \\
\text { y asesoramiento que alivie el } \\
\text { sufrimiento de los familiares. }\end{array}$ \\
\hline $\begin{array}{l}\text { Abrir la } \\
\text { unidad de } \\
\text { cuidados } \\
\text { intensivos a } \\
\text { los } \\
\text { familiares: } \\
\text { ¿qué opinan } \\
\text { los } \\
\text { profesionale } \\
\text { s? }\end{array}$ & $\begin{array}{l}\text { C. Zaforteza } \\
\text { Lallemand, A. } \\
\text { García Mozo, } \\
\text { R. Quintana } \\
\text { Carbonero, C. } \\
\text { Sánchez } \\
\text { Calvín, E. } \\
\text { Abadía Ortiz y } \\
\text { J. Albert Miró } \\
\text { Gayà. }\end{array}$ & 2010 & $\begin{array}{l}\text { Enfermer } \\
\text { ía } \\
\text { intensiva }\end{array}$ & $\begin{array}{l}\text { Metodolog } \\
\text { ía } \\
\text { cualitativa: } \\
\text { diseño } \\
\text { investigaci } \\
\text { ón- acción } \\
\text { participati } \\
\text { va }\end{array}$ & $\begin{array}{l}\text { Los profesionales de la UCI son } \\
\text { reticentes a la apertura de la unidad, } \\
\text { pero admiten ampliar los horarios de } \\
\text { visita actuales, ya que la estructura } \\
\text { física de la unidad es una barrera } \\
\text { importante. }\end{array}$ \\
\hline $\begin{array}{l}\text { Desarrollo } \\
\text { de una guía } \\
\text { de atención } \\
\text { a los } \\
\text { familiares } \\
\text { del paciente } \\
\text { crítico }\end{array}$ & $\begin{array}{l}\text { A. García } \\
\text { Mozo, F. } \\
\text { Sánchez } \\
\text { Roldán, S.M. } \\
\text { Amorós Cerdá, } \\
\text { F. Balaguer } \\
\text { Albarracín, M. } \\
\text { Díez Alcalde, } \\
\text { M.T. Durán } \\
\text { Torres et al. }\end{array}$ & 2010 & $\begin{array}{l}\text { Enfermer } \\
\text { ía } \\
\text { intensiva }\end{array}$ & $\begin{array}{l}\text { Metodolog } \\
\text { ía } \\
\text { cualitativa: } \\
\text { diseño } \\
\text { investigaci } \\
\text { ón- acción } \\
\text { participati } \\
\text { va }\end{array}$ & $\begin{array}{l}\text { Se ha desarrollado una guía de } \\
\text { atención a los familiares de la UCI que } \\
\text { es una herramienta útil para dar } \\
\text { seguridad al profesional y unificar los } \\
\text { criterios de actuación. }\end{array}$ \\
\hline $\begin{array}{l}\text { Mejorando } \\
\text { el cuidado a } \\
\text { los } \\
\text { familiares } \\
\text { del paciente } \\
\text { crítico: } \\
\text { estrategias } \\
\text { consensuad } \\
\text { as }\end{array}$ & $\begin{array}{l}\text { C. Zaforteza } \\
\text { Lallemand, S. } \\
\text { Prieto } \\
\text { González, T.P. } \\
\text { Canet Ferrer, } \\
\text { Y. Díaz López, } \\
\text { M. Molina } \\
\text { Santiago, C. } \\
\text { Moreno Mulet } \\
\text { et al. }\end{array}$ & 2010 & $\begin{array}{l}\text { Enfermer } \\
\text { ía } \\
\text { intensiva }\end{array}$ & $\begin{array}{l}\text { Metodolog } \\
\text { ía } \\
\text { cualitativa: } \\
\text { diseño } \\
\text { investigaci } \\
\text { ón- acción } \\
\text { participati } \\
\text { va }\end{array}$ & $\begin{array}{l}\text { Se elaboraron } 2 \text { documentos: 1) } \\
\text { diagnóstico de situación en el que se } \\
\text { describe la situación actual de la } \\
\text { atención de los familiares y 2) } 10 \\
\text { propuestas de cambio, de las que se } \\
\text { priorizaron } 5 \text {, las cuales son: difusión } \\
\text { entre los profesionales de la evidencia } \\
\text { sobre familiares en la UCl a través de } \\
\text { una sesión y la entrega de trípticos } \\
\text { informativos, mejora de los procesos } \\
\text { de información (enfermera-familia e } \\
\text { intraequipo). }\end{array}$ \\
\hline
\end{tabular}




\begin{tabular}{|c|c|c|c|c|c|}
\hline $\begin{array}{l}\text { Care and } \\
\text { Caring in } \\
\text { the } \\
\text { intensive } \\
\text { care unit: } \\
\text { Family } \\
\text { members' } \\
\text { distress and } \\
\text { perceptions } \\
\text { about staff } \\
\text { skills, } \\
\text { communicat } \\
\text { ion, and } \\
\text { emotional } \\
\text { support. }\end{array}$ & $\begin{array}{l}\text { Eve B. } \\
\text { Carlson, David } \\
\text { A. Spain, Luma } \\
\text { Muhtadie, Liz } \\
\text { McDade- } \\
\text { Montez and } \\
\text { Kathryn S. } \\
\text { Macia }\end{array}$ & 2015 & $\begin{array}{l}\text { Journal } \\
\text { of Critical } \\
\text { Care }\end{array}$ & $\begin{array}{l}\text { Estudio } \\
\text { cuantitativ } \\
\text { o } \\
\text { longitudin } \\
\text { al }\end{array}$ & $\begin{array}{l}\text { Una mayor comprensión de los efectos } \\
\text { del estrés traumático en la familia } \\
\text { puede ayudar al personal de la UCl a } \\
\text { mejorar la comunicación y aumentar la } \\
\text { satisfacción de la familia. }\end{array}$ \\
\hline $\begin{array}{l}\text { The } \\
\text { experiences } \\
\text { of nurse in } \\
\text { providing } \\
\text { psychosocia } \\
\text { I support to } \\
\text { families of } \\
\text { critically ill } \\
\text { trauma } \\
\text { patients in } \\
\text { intensive } \\
\text { care units. A } \\
\text { study in the } \\
\text { Durban } \\
\text { metropolitan } \\
\text { area }\end{array}$ & $\begin{array}{l}\text { P Brysiewicz } \\
\text { and B R } \\
\text { Bhengu }\end{array}$ & 2010 & $\begin{array}{l}\text { Southern } \\
\text { African } \\
\text { Journal } \\
\text { of Critical } \\
\text { Care } \\
\text { (SAJCC) }\end{array}$ & $\begin{array}{l}\text { Estudio } \\
\text { cualitativo: } \\
\text { enfoque } \\
\text { fenomenol } \\
\text { ógico }\end{array}$ & $\begin{array}{l}\text { La mayoría de las enfermeras de este } \\
\text { estudio sintieron que cuatro factores } \\
\text { principales afectan en el apoyo } \\
\text { psicosocial que puedan prestar a las } \\
\text { familias de los pacientes críticos } \\
\text { ingresados en la Unidad de Cuidados } \\
\text { Intensivos, estos factores son: } \\
\text { diversidad cultural, barreras de } \\
\text { comunicación, recursos insuficientes y } \\
\text { la formación del personal insuficiente. }\end{array}$ \\
\hline $\begin{array}{l}\text { Involving } \\
\text { relatives in } \\
\text { ICU patient } \\
\text { care: critical } \\
\text { care } \\
\text { Nursing } \\
\text { challenges }\end{array}$ & $\begin{array}{l}\text { Bridget } \\
\text { McConnell and } \\
\text { Tracey } \\
\text { Moroney }\end{array}$ & 2015 & $\begin{array}{l}\text { Journal } \\
\text { of } \\
\text { Clinical } \\
\text { Nursing } \\
\text { (JCN) }\end{array}$ & $\begin{array}{l}\text { Diseño } \\
\text { mixto: } \\
\text { fase } 1 \\
\text { cuantitativ } \\
\text { a y fase } 2 \\
\text { cualitativa. }\end{array}$ & $\begin{array}{l}\text { Se identificaron algunas barreras en la } \\
\text { participación de las familias en el } \\
\text { cuidado del paciente crítico, así, los } \\
\text { factores relacionados con el paciente } \\
\text { crítico, la UCI, la enfermera de } \\
\text { Cuidados Intensivos y el medio de la } \\
\text { UCl dificultan esta participación. } \\
\text { Además, este estudio ha identificado } \\
\text { que, al considerar la participación } \\
\text { familiar en la atención del paciente, las } \\
\text { enfermeras asumen un papel } \\
\text { paternalista. }\end{array}$ \\
\hline $\begin{array}{l}\text { Aspects that } \\
\text { facilitate or } \\
\text { interfere in } \\
\text { the } \\
\text { communicat } \\
\text { ion process } \\
\text { between } \\
\text { nursing } \\
\text { professional } \\
\text { s and } \\
\text { patients in } \\
\text { critical state }\end{array}$ & $\begin{array}{l}\text { Diana Marcela } \\
\text { Achury } \\
\text { Saldaña, } \\
\text { Maribel Pinilla } \\
\text { Alarcón and } \\
\text { Herly Alvarado } \\
\text { Romero }\end{array}$ & 2015 & $\begin{array}{l}\text { Investiga } \\
\text { ción y } \\
\text { Educació } \\
\text { n en } \\
\text { Enfermer } \\
\text { ía }\end{array}$ & $\begin{array}{l}\text { Estudio } \\
\text { cuantitativ } \\
\text { o } \\
\text { descriptiv } \\
\text { o }\end{array}$ & $\begin{array}{l}\text { El } 91,6 \% \text { de los profesionales de } \\
\text { enfermería considera importante la } \\
\text { comunicación con el paciente y su } \\
\text { familia; el } 75,9 \% \text { intenta proporcionar } \\
\text { cuidado físico y comunicarse por turno } \\
\text { con el paciente y su familia; el } 50 \% \\
\text { refiere sentir temor para comunicarse y } \\
\text { tan solo el } 53,7 \% \text { integra las } \\
\text { emociones en el cuidado físico del } \\
\text { paciente. El proceso comunicativo } \\
\text { entre la enfermera y el paciente de } \\
\text { UCl está limitado por las políticas } \\
\text { restrictivas. }\end{array}$ \\
\hline
\end{tabular}




\begin{tabular}{|c|c|c|c|c|c|}
\hline $\begin{array}{l}\text { Meeting the } \\
\text { needs of } \\
\text { patients' } \\
\text { families in } \\
\text { intensive } \\
\text { care units }\end{array}$ & Rabia Khalaila & 2014 & $\begin{array}{l}\text { Nursing } \\
\text { standard }\end{array}$ & $\begin{array}{l}\text { Revisión } \\
\text { de } \\
\text { artículos }\end{array}$ & $\begin{array}{l}\text { Los resultados de estos estudios } \\
\text { revelaron que proporcionar a las } \\
\text { familias estrategias de comunicación e } \\
\text { información, desarrollar programas de } \\
\text { educación y establecer una atención } \\
\text { centrada en la familia puede ser eficaz } \\
\text { para aumentar la satisfacción de los } \\
\text { familiares. }\end{array}$ \\
\hline $\begin{array}{l}\text { How do } \\
\text { intensive } \\
\text { care nurses } \\
\text { perceive } \\
\text { families in } \\
\text { intensive } \\
\text { care? } \\
\text { Insights } \\
\text { from the } \\
\text { United } \\
\text { Kingdom } \\
\text { and } \\
\text { Australia }\end{array}$ & $\begin{array}{l}\text { Susanne Kean } \\
\text { and Marion } \\
\text { Mitchell }\end{array}$ & 2013 & $\begin{array}{l}\text { Journal } \\
\text { of } \\
\text { Clinical } \\
\text { Nursing } \\
\text { (JCN) }\end{array}$ & $\begin{array}{l}\text { Estudio } \\
\text { cuantitativ } \\
\text { o } \\
\text { cuasi- } \\
\text { experimen } \\
\text { tal }\end{array}$ & $\begin{array}{l}\text { Las enfermeras han llegado a la } \\
\text { conclusión de que invitar a la familia a } \\
\text { ser parte de la atención del paciente } \\
\text { debe ser una práctica diaria en estas } \\
\text { unidades, ya que las familias son el } \\
\text { recurso en el cuidado de estos } \\
\text { pacientes durante una recuperación a } \\
\text { menudo prolongada. }\end{array}$ \\
\hline $\begin{array}{l}\text { Half of the } \\
\text { family } \\
\text { members of } \\
\text { critically ill } \\
\text { patients } \\
\text { experience } \\
\text { excessive } \\
\text { daytime } \\
\text { sleepiness }\end{array}$ & $\begin{array}{l}\text { Avelino C. } \\
\text { Verceles, } \\
\text { Douglas S. } \\
\text { Corwin, Majid } \\
\text { Afshar, Eliot B. } \\
\text { Friedman, } \\
\text { Michael T. } \\
\text { McCurdy, Carl } \\
\text { Shanholtz, } \\
\text { Karen } \\
\text { Oakjones et al. }\end{array}$ & 2014 & $\begin{array}{l}\text { Intensive } \\
\text { Care } \\
\text { Med }\end{array}$ & $\begin{array}{l}\text { Estudio } \\
\text { cuantitativ } \\
\text { o } \\
\text { observaci } \\
\text { onal } \\
\text { descriptiv } \\
\text { o }\end{array}$ & $\begin{array}{l}\text { La mitad de los miembros de la familia } \\
\text { de los pacientes de la Unidad de } \\
\text { Cuidados Intensivos experimentan un } \\
\text { mayor deterioro en el desempeño de } \\
\text { las actividades diarias, pero no } \\
\text { padecen de embotamiento cognitivo. }\end{array}$ \\
\hline $\begin{array}{l}\text { Patients' } \\
\text { and Health } \\
\text { Care } \\
\text { Providers' } \\
\text { Perception } \\
\text { of Stressors } \\
\text { in the } \\
\text { Intensive } \\
\text { Care Units }\end{array}$ & Alham Abuatiq & 2015 & $\begin{array}{l}\text { Dimensio } \\
\text { ns of } \\
\text { Critical } \\
\text { Care } \\
\text { Nursing }\end{array}$ & $\begin{array}{l}\text { Diseño } \\
\text { mixto: } \\
\text { diseño } \\
\text { descriptiv } \\
\text { o- } \\
\text { comparati } \\
\text { vo. } \\
\text { y diseño } \\
\text { fenomenol } \\
\text { ógico }\end{array}$ & $\begin{array}{l}\text { Los tres ítems más estresantes } \\
\text { percibidos por los pacientes son: } \\
\text { "sentir dolor", seguido de "no ser capaz } \\
\text { de dormir" y "preocupaciones } \\
\text { financieras". Según los profesionales } \\
\text { el ranking es "sentir dolor", "no ser } \\
\text { capaz de comunicarse" y "no tener el } \\
\text { control de sí mismo" como los } 3 \\
\text { factores estresantes percibidos por sus } \\
\text { pacientes. } \\
\text { Esta información puede contribuir a las } \\
\text { valoraciones de enfermería y al } \\
\text { cuidado del paciente, además puede } \\
\text { animar al personal de la UCI a } \\
\text { rediseñar el ambiente de la UCI para } \\
\text { ser menos estresante. }\end{array}$ \\
\hline
\end{tabular}




\begin{tabular}{|c|c|c|c|c|c|}
\hline $\begin{array}{l}\text { Multidiscipli } \\
\text { nary Team } \\
\text { Training to } \\
\text { Enhance } \\
\text { Family } \\
\text { Communica } \\
\text { tion in the } \\
\text { ICU }\end{array}$ & $\begin{array}{l}\text { David J. Shaw, } \\
\text { Judy E. } \\
\text { Davidson, } \\
\text { Renée I. } \\
\text { Smilde, Tarane } \\
\text { Sondoozi and } \\
\text { Donna Agan. }\end{array}$ & 2013 & $\begin{array}{l}\text { Critical } \\
\text { Care } \\
\text { Medicine }\end{array}$ & $\begin{array}{l}\text { Diseño } \\
\text { pre- } \\
\text { intervenci } \\
\text { ón y } \\
\text { postinterv } \\
\text { ención }\end{array}$ & $\begin{array}{l}\text { Una sesión de entrenamiento } \\
\text { interactivo de } 90 \text { minutos, junto con } \\
\text { una prelectura, una lista de } \\
\text { comentarios actuales de los familiares } \\
\text { de la UCl y un caso simulado que } \\
\text { involucra a todos los miembros que } \\
\text { participaban en la formación, mejora la } \\
\text { satisfacción familiar con muchas áreas } \\
\text { claves de la toma de decisiones en la } \\
\text { UCl que está relacionado con la } \\
\text { comunicación. }\end{array}$ \\
\hline $\begin{array}{l}\text { Relatives' } \\
\text { perspective } \\
\text { s on the } \\
\text { quality of } \\
\text { care in an } \\
\text { Intensive } \\
\text { Care Unit: } \\
\text { The } \\
\text { theoretical } \\
\text { concept of a } \\
\text { new tool }\end{array}$ & $\begin{array}{l}\text { Margo M.C. } \\
\text { van Mol, } \\
\text { Esther C. } \\
\text { Bakker, Marjan } \\
\text { D. Nijkamp, } \\
\text { Erwin J.O. } \\
\text { Kompanje, Jan } \\
\text { Bakker and } \\
\text { Lisbeth } \\
\text { Verharen }\end{array}$ & 2014 & $\begin{array}{l}\text { Patient } \\
\text { Educatio } \\
\text { n and } \\
\text { Counseli } \\
\text { ng (PEC) }\end{array}$ & $\begin{array}{l}\text { Estudio } \\
\text { cuantitativ } \\
\text { o }\end{array}$ & $\begin{array}{l}\text { El Índice de Calidad del Consumidor } \\
\text { "Los Familiares de Unidad de } \\
\text { Cuidados Intensivos" (CQI-“ R-ICU”) } \\
\text { es un instrumento válido mediante el } \\
\text { cual los profesionales pueden } \\
\text { aprender de las experiencias de los } \\
\text { familiares y esto puede proporcionar } \\
\text { información sobre los puntos a } \\
\text { mejorar. }\end{array}$ \\
\hline $\begin{array}{l}\text { Resolving } \\
\text { Communica } \\
\text { tion } \\
\text { Challenges } \\
\text { in the } \\
\text { Intensive } \\
\text { Care Unit }\end{array}$ & Marian Grant & 2015 & $\begin{array}{l}\text { American } \\
\text { Associati } \\
\text { on of } \\
\text { Critical- } \\
\text { Care } \\
\text { Nurses } \\
\text { (AACN) } \\
\text { Advance } \\
\text { d Critical } \\
\text { Care }\end{array}$ & $\begin{array}{l}\text { Revisión } \\
\text { de } \\
\text { artículos }\end{array}$ & $\begin{array}{l}\text { Existen tres tipos de problemas de } \\
\text { comunicación que se encuentran en } \\
\text { esta unidad: los que están dentro del } \\
\text { equipo de la UCl, los que existen entre } \\
\text { el equipo de la UCl y el paciente o la } \\
\text { familia, y los que están dentro de la } \\
\text { familia del paciente. }\end{array}$ \\
\hline $\begin{array}{l}\text { Writing In } \\
\text { and } \\
\text { Reading } \\
\text { ICU Diaries: } \\
\text { Qualitative } \\
\text { Study of } \\
\text { Families' } \\
\text { Experience } \\
\text { in the ICU }\end{array}$ & $\begin{array}{l}\text { Maité } \\
\text { Garraouste- } \\
\text { Orgeas, } \\
\text { Antoine Périer, } \\
\text { Philippe } \\
\text { Mouricou, } \\
\text { Charles } \\
\text { Grégoire, } \\
\text { Cédric Bruel, } \\
\text { Sandie } \\
\text { Brochon et al. }\end{array}$ & 2014 & $\begin{array}{l}\text { PLoS } \\
\text { ONE }\end{array}$ & $\begin{array}{l}\text { Estudio } \\
\text { cualitativo: } \\
\text { teoría } \\
\text { fundament } \\
\text { ada }\end{array}$ & $\begin{array}{l}\text { Tras investigar la experiencia de las } \\
\text { familias con la lectura y la escritura en } \\
\text { los diarios de los pacientes ingresados } \\
\text { en la UCI, los resultados indican } \\
\text { efectos positivos de los diarios sobre } \\
\text { los miembros de la familia del paciente } \\
\text { crítico. }\end{array}$ \\
\hline $\begin{array}{l}\text { Diaries for } \\
\text { recovery } \\
\text { from critical } \\
\text { illness } \\
\text { (Review) }\end{array}$ & $\begin{array}{l}\text { Amanda J } \\
\text { Ullman, } \\
\text { Leanne M } \\
\text { Aitken, Janice } \\
\text { Rattray, Justin } \\
\text { Kenardy, } \\
\text { Robyne Le } \\
\text { Brocque, } \\
\text { Stephen } \\
\text { MacGillivray et } \\
\text { al. }\end{array}$ & 2014 & $\begin{array}{l}\text { The } \\
\text { Cochran } \\
\text { e } \\
\text { Collabora } \\
\text { tion }\end{array}$ & $\begin{array}{l}\text { Revisión } \\
\text { de } \\
\text { artículos }\end{array}$ & $\begin{array}{l}\text { Actualmente no hay evidencia } \\
\text { suficiente de los beneficios o perjuicios } \\
\text { de los diarios para los pacientes } \\
\text { ingresados en la Unidad de Cuidados } \\
\text { Intensivos como para sus familiares, } \\
\text { aunque un estudio ha descrito que } \\
\text { estos diarios reducen la sintomatología } \\
\text { de estrés post-traumático en los } \\
\text { miembros de la familia. }\end{array}$ \\
\hline
\end{tabular}



APOYO EMOCIONAL DE LAS FAMILIAS A LOS PACIENTES EN UNIDADES DE CUIDADOS INTENSIVOS

\begin{tabular}{|c|c|c|c|c|c|}
\hline $\begin{array}{l}\text { Revisión de } \\
\text { los estudios } \\
\text { sobre los } \\
\text { profesionale } \\
\text { s de } \\
\text { enfermería } \\
\text { de las } \\
\text { Unidades } \\
\text { de } \\
\text { Cuidados } \\
\text { Intensivos } \\
\text { de España }\end{array}$ & $\begin{array}{l}\text { Jesús Manuel } \\
\text { Navarro } \\
\text { Arnedo }\end{array}$ & 2012 & $\begin{array}{l}\text { Enfermer } \\
\text { ía global }\end{array}$ & $\begin{array}{l}\text { Revisión } \\
\text { de } \\
\text { artículos }\end{array}$ & $\begin{array}{l}\text { Las enfermeras afirmaban que el } \\
\text { trabajo en una Unidad de Cuidados } \\
\text { Intensivos era estresante debido al } \\
\text { estado de gravedad de los pacientes y } \\
\text { al entorno laboral. Opinaban, tras } \\
\text { modificar las normas de visita, que la } \\
\text { familia proporcionaba apoyo emocional } \\
\text { al paciente e incrementaba su deseo } \\
\text { de vivir. Además, reconoce que es } \\
\text { necesario una preparación específica } \\
\text { para dar apoyo emocional al paciente } \\
\text { y su familia. }\end{array}$ \\
\hline
\end{tabular}

